\title{
La retórica emocional de la esposa del candidato: análisis lingüístico de los discursos de Michelle Obama y Ann Romney
}

\author{
Rocío ZAMORA MEDINA \\ Universidad de Murcia \\ rzamoramedina@um.es \\ Celia Berná Sicilia \\ Universidad Católica San Antonio de Murcia \\ cberna@ucam.edu \\ Helena MARTÍNEZ MARTÍNEZ \\ Universidad Católica San Antonio de Murcia \\ hmmartinez@ucam.edu
}

Recibido: 26 de junio de 2013

Aceptado: 19 de noviembre de 2013

\begin{abstract}
Resumen
Esta investigación se centra en la construcción del relato emocional a través del análisis textual de los discursos pronunciados por las candidatas a Primera Dama, Ann Romney y Michelle Obama en las convenciones republicana y demócrata de la campaña presidencial de Estados Unidos de 2012. El principal objetivo consiste en identificar los marcadores emocionales presentes en los textos pronunciados respectivamente por cada una en dichas convenciones y que afectan al nivel semántico, morfosintáctico y pragmático. El análisis de los recursos lingüísticos utilizados en cada texto desvela que, como pieza clave de la estrategia de la campaña, ambas articularon un relato intencionadamente despolitizado y con una marcada orientación emocional, focalizando la atención en la dimensión humana de sus respectivos maridos, lo que contribuyó a dibujar un perfil más personal e íntimo del candidato.

Palabras clave: emoción, Primera Dama, análisis del discurso, comunicación política, campaña electoral USA.

\section{Emotional rhetoric of candidate's wife: linguistic analysis of the speeches of Michelle Obama and Ann Romney}

\begin{abstract}
This research focuses on the emotional story building process based on the textual analysis of candidates for First Lady, Ann Romney and Michelle Obama, speeches that were pronounced during the Republican and Democratic conventions of the U.S. presidential campaign of 2012. The main objective is to identify the emotional markers included in the texts delivered respectively in each of these conventions that affect the semantic, morphosyntactic and pragmatic level. The analysis of the linguistic resources used reveals that, as a key part of the electoral campaign strategy, both were deliberately articulated as a non political speech, and sustained a marked emotional storytelling, focusing attention on the human dimension of their husbands, a fact that contributed to draw a more personal and intimate of the candidate profile.

Keywords: emotion, First Lady, discourse analysis, political communication, USA electoral campaign.

\section{Referencia normalizada}

ZAMORA MEDINA, Rocío; BERNÁ SICILIA, Celia; y MARTÍNEZ MARTÍNEZ, Helena (2014): “La retórica emocional de la esposa del candidato: análisis lingüístico de los discursos de Michelle Obama y Ann Romney". Estudios sobre el Mensaje Periodístico. Vol. 20, Núm. 1 (enero-junio), págs.: 585-612. Madrid, Servicio de Publicaciones de la Universidad Complutense.
\end{abstract}


Sumario: 1. Introducción. 2. La importancia del discurso emocional como estrategia discursiva en la comunicación política. 3. La Primera Dama como recurso humanizador de la estrategia de campaña del candidato 4. Método 5. Análisis y Resultados; 5.1. Recursos semánticos utilizados en la construcción del relato emocional; 5. 2. Recursos morfosintácticos utilizados en la construcción del relato emocional; 5.3. Recursos pragmáticos utilizados en la construcción del relato emocional; 5.4. La construcción del perfil humano del candidato presidencial: atributos y valores; 5.5. Valoración del seguimiento y la resonancia de ambos discursos en las redes sociales. 6. Conclusiones 7. Referencias bibliográficas. 8. Anexo 1: Discursos de Ann Romney y Michelle Obama; 8.1. Discurso íntegro de Ann Romney en Tampa; 8. 2. Discurso íntegro de Michelle Obama en Charlotte. 9. Anexo 2: Campos semánticos en los discursos de Ann Romney y Michelle Obama: unidades léxicas y frecuencias.

\section{Introducción}

La figura de la esposa del candidato ha ido adquiriendo un protagonismo cada vez mayor dentro de las campañas presidenciales de Estados Unidos. Su papel a la hora de desvelar el lado más emocional del liderazgo político se ha revelado clave en la estrategia por la carrera electoral, dada su influencia sobre la imagen pública que los aspirantes a la presidencia proyectan en los electores (Burns, 2005).

En las elecciones de 2012, caracterizadas por una intensa personalización en la estrategia política electoral, Ann Romney y Michelle Obama ejercieron de portavoces de excepción de los valores como personas y como hombres de familia de sus respectivos maridos, por lo que fueron consideradas por el ciudadano corriente, acostumbrado al abismo insalvable que en ocasiones separa la política de la realidad (Arroyas, Pérez y Berná, 2012), como representantes de lo auténtico y lo mundano.

En el contexto de las convenciones demócrata y republicana, el afán por mostrar la conexión entre política y emoción se hizo, si cabe, más evidente. Asumiendo la tarea de ofrecer un perfil personal e intimista de los candidatos, las aspirantes a Primeras Damas construyeron un relato "humanizante" que buscaba esencialmente la complicidad y la empatía del público y, para ello, se valieron de un arma poderosa: el lenguaje.

Este trabajo desvela las claves del lenguaje emocional utilizado por las esposas del candidato a través de un estudio pormenorizado y sistemático de los recursos lingüísticos puestos al servicio de la argumentación emocional dentro de los discursos pronunciados por Michelle Obama en Charlotte y por Ann Romney en Tampa (Anexo 1). La comparativa entre ambas alocuciones atiende a distintos niveles de análisis -léxico-semántico, morfosintáctico y pragmático- y se ha realizado a partir de la información obtenida dese herramientas textuales cuantitativas como Wordle.net y Textalyser y gracias a la aplicación de técnicas cualitativas de análisis del discurso.

\section{La importancia del discurso emocional como estrategia discursiva en la co- municación política}

Las emociones han sido objeto de diversas aproximaciones en el ámbito teórico desde el campo de la psicología, la sociología o la publicidad, aunque especial interés han despertado en el marco de la comunicación política, la lingüística, la teoría de la argumentación y el análisis del discurso.

Son muchos quienes en el campo de la investigación política han puesto de manifiesto la importancia de la argumentación emocional en la estrategia política. Diferentes estudios desde el ámbito de la neuropsicología, han demostrado la importancia que las emociones tienen en la recepción y aceptación de los discursos y los efectos 
que generan en los oyentes (Westen, 2007). En esta línea, Luntz sitúa las emociones como una de las claves que propician esa recepción y comprensión del lenguaje y asegura que lo importante no es lo que se dice, sino lo que la gente entiende. Por este motivo, según dicho autor, ha de conferirse mayor relevancia al contexto que al propio mensaje, por brillante y racional que este resulte (Luntz, 2007: 18).

Además, también hay un buen número de trabajos que, desde las teorías sobre el liderazgo, apuntan a un uso cada vez más frecuente de las emociones en el discurso político (Zaccaro, 2012; Rajah, Song, y Arvey, 2011). Desde los clásicos, todo buen orador ha sido consciente de la importancia que tenía evocar en su discurso diversos sentimientos y emociones para persuadir a su auditorio. En este sentido, Redlawsk (2006: 1) señala "the central role of passion in politics" y evidencia que los sentimientos de la gente adquieren una relevancia esencial en los cálculos políticos, fundamentalmente, porque nuestras emociones influyen de modo decisivo en nuestra forma de pensar y en el modo que tenemos de procesar la información.

Hofinger y Manz-Crist se sitúan en la misma línea y conceden a las emociones un rol principal en la estrategia política:

It seems that, at some point, we need to feel "That's right" in order to find our preferred party or candidate. As already mentioned, finding the right solution for a tricky problem is easier and better, if we give our unconscious mind some time to work out the decision. And it is safe to assume that, during the unconscious decision processing, emotions also play their part. (Honfinger, Manz-Crist, 2011)

Morgante, por su parte, también sostiene que la emoción constituye un componente esencial del discurso político y advierte de la estrecha interrelación que existe entre comunicación, política y pasión:

De ce point de vue, communication, politique et passion sont donc à voir comme trois variables liées à autant de dimensions conceptuelles dont le croisement peut être envisagé comme le lieu d'une réflexion qui, tout en s'appuyant sur la conscience depuis longtemps acquise de leur étroite interaction, peut devenir aussi un instrument pour mieux analyser notre modernité. (Morgante, 2010: 18-19).

Por otro lado, los estudios en torno a la relación que vincula la lengua con las pasiones se han incrementado, a su vez, significativamente en los últimos años (Puig, 2008: 393). La propagación de las perspectivas interaccionistas dentro de la reflexión lingüística propició que los investigadores prestaran una atención preferente al lenguaje emotivo, lenguaje que ya no fue considerado como un elemento perteneciente única y exclusivamente a la esfera de la experiencia íntima del sujeto, sino que fue descrito en su dimensión comunicativa tomando también en consideración cuál es la reacción que el mensaje desencadena en el destinatario (Kerbrat-Orecchioni, 2000: 50)1.

1 Tal como evidencia Kerbrat-Orecchioni (2000: 20) en su reflexión sobre las categorías afectivas en la lengua y el discurso, la interactividad constituye una propiedad incuestionable de la emotividad en el lenguaje. De acuerdo con esta autora, la emoción no se vincula solo con el hablante, sino que establece con el alocutario del mensaje una interacción fundamental: el receptor desencadena un proceso empático con el locutor que le revela sus pasiones gracias a la activación del "efecto patémico". 
La consideración de las emociones como elemento crucial en los procesos persuasivos ha hecho que, en los últimos años, los asesores políticos se hayan centrado no solo en los candidatos a la hora de diseñar las estrategias políticas y electorales, sino también en sus esposas e hijos. Este giro ha propiciado que los discursos políticos en muchas ocasiones dejaran en un segundo plano el programa electoral como tal y apelaran directamente a las pasiones, pues estas "se reconocen como un factor fundamental de la racionalidad y de la interacción entre los seres humanos" (Puig, 2008: 412).

Debido a la gran importancia que se ha concedido a la parte emocional como recurso fundamental de la estrategia de campaña y el marketing político, otros autores recuerdan la necesidad de tener en cuenta que la importancia de las emociones no es sinónimo siempre de buenos resultados, si no que pueden ser utilizadas también de modo negativo:

Nonetheless, it is important to recognize that manipulation is not unique to emotions. Any political tactic can be used in a disingenuous or deceptive manner. Contemporary science has been right to redeem the importance of emotion, but we should not make the opposite mistake by assuming that emotions are always forces for good outcomes. On our own or with the "help" of others, we can react with too much fear, too much anger, or too much enthusiasm, and these emotions can at times lead us astray just as they can help us- in our political lives as well as our lives more broadly. (Brader, 2011: 52).

\section{La Primera Dama como recurso humanizador de la estrategia de campaña del candidato}

A diferencia de otros países, caracterizados por un cultura política en la que la vida privada queda eclipsada a la visibilidad mediática, en la política norteamericana, marcada por una fuerte cultura de la personalización, la presencia de la familia y, en especial, de la Primera Dama puede constituir una fuerza política determinante en la construcción de la imagen de candidato, así como en la movilización de los votantes durante la campaña electoral (VanHorn, 2010).

En las campañas electorales americanas es corriente el recurso a los surrogates o personas próximas al candidato, como es el caso de la esposa del candidato, que intervienen en su nombre y le sustituyen en muchos actos. El objetivo de sus intervenciones, en última instancia, es proporcionar a los electores información sobre el candidato, especialmente buscando proyectar ese carácter personal del futuro presidente con el que la mayoría de los ciudadanos pueda sentirse identificado. De este modo, la implicación personal de la esposa del candidato forma parte de la estrategia integral de la campaña (MacManus y Quecan, 2008), y tiene como objetivo proyectar el lado más emocional del liderazgo político e influir así sobre la imagen pública del candidato (Burns, 2005).

Tal y como algunos trabajos han apuntado, el papel de la Primera Dama ha evolucionado considerablemente (Watson, 2000). De ser meros apoyos complementarios y fieles acompañantes del líder del país, han pasado a asumir un papel más activo, involucrándose cada vez más en la estrategia de la campaña. Sin embargo, a pesar de la intensificación de su papel en la actividad política presidencial, existe un debate abierto en torno al papel que éstas deben asumir en relación al grado de su implica- 
ción política. Y es que, como apunta Winfield (2000), "cuanto más activa es políticamente una Primera Dama, más cobertura negativa recibe por parte de los medios de comunicación".

En las elecciones presidenciales de 2012, concretamente, las esposas de los candidatos se convirtieron en su mayor activo político y las más files abogadas de sus valores como personas y hombres de familia (Gutiérrez-Rubí, 2012). Hasta tal punto que se les exigió una implicación personal y emocional en la campaña sin precedentes. Su papel fue trascendental para ofrecer un valor añadido, y a la vez diferencial, de la candidatura de sus respectivos esposos, sobre todo en un contexto electoral en el que el electorado femenino podía resultar trascendental.

En estas ocasiones, los discursos de la Primera Dama concentran toda la atención pública y mediática. A través del relato de su matrimonio y su vida familiar, ambas se enfrentaban al desafío y la dificultad de canalizar la verbalización y las acciones espontáneas en formas apropiadas para el ámbito público (Wertheimer, 2005). En este sentido, sus habilidades de comunicación suelen determinar la valoración de sus intervenciones por parte de los medios de comunicación (Caroli, 2003).

La misión reservada a ambas esposas en sus respectivos discursos consistía en contar en primera persona y desde una perspectiva íntima quién era el candidato, para ofrecer un retrato que lo humanizara y lo acercara más a la ciudadanía. Como apunta Gutiérrez-Rubí (2012: 232), en medio de una dura batalla electoral, "sus parejas, al final, se convierten en ese único enlace con lo terrenal, con lo mundano, con lo accesible para el ciudadano corriente". Se trata de "ofrecer una ventana para descubrir el carácter del candidato" (Grimes, 1990:16). Incluso, su implicación también puede suponer un impulso para conseguir que se registren más mujeres para votar, empujarles a ir a las urnas y, de esta forma, estrechar las brechas de género (Watson, 2000).

En última instancia, a través de este tipo de intervenciones, se espera que esas mujeres puedan dar testimonio del auténtico hombre que hay detrás del candidato, de que su intimidad o su vida privada -como marido, padre y gestor de los asuntos familiares- sirva para ilustrar su moralidad y su auténtico carácter. En este sentido, el desafío radica en hacer ver que, detrás del hombre que aspira a ser la persona con más poder del mundo, hay un ser humano con sus fortalezas y debilidades. En definitiva, "intentar conectar con el electorado y demostrar, a través de sus relaciones con los candidatos, que las razones por las cuales se habían enamorado de ellos eran las mismas por las que la gente debía votarles" (Gutiérrez-Rubí, 2012: 284 y ss.).

Así, la proyección pública de la propia biografía de la Primera Dama a lo largo de toda la campaña, y especialmente durante el momento estelar que supone la convención nacional, se convierte en pieza clave de su implicación en la campaña. Es una tendencia cada vez mayor que ésta utilice su propia imagen para crear la imagen de su marido, alabando aquellos atributos de su pareja más valorados por los votantes. En esta ocasión, Michelle Obama se presentó como madre y esposa, pero también como socia, aliada, cómplice y parte del equipo presidencial. Ann Romney asumía con orgullo su papel de ama de casa y educadora de sus cinco hijos, un trabajo muy digno en su opinión, alejándose así de las críticas lanzadas desde el partido demócrata que le acusaron de no haber trabajado ni un sólo día en su vida. 
Uno de los recursos más utilizados para humanizar la figura del candidato es, sin duda, alejarse, en primer lugar, de todo lo que suene a la política dura, y dar paso a un discurso político "suavizado" basado en el relato emocional que tan eficaz resulta para conectar con el electorado. En este caso concreto, en ambos discursos se activaron tácticas retóricas afectivas puestas en práctica al servicio de unos determinados intereses políticos. De hecho, las dos esposas hicieron explícita, en varias ocasiones, la supuesta despolitización de su discurso, apuntando a una marcada orientación emocional del mismo. Así, Ann Romney, declaró: "I want to talk to you tonight not about politics and not about party [...] I want to talk to you from my heart about our hearts". También más adelante señala "I want to talk to you about love". Por su parte, para Michelle Obama, las experiencias personales y los valores de Barack incidían decisivamente en sus actitudes y decisiones políticas, lo que hacía de él un candidato empático que entiende los problemas y preocupaciones de la ciudadanía: "For Barack, these issues aren't political, they're personal".

El análisis empírico de los discursos pronunciados en sus esposas en las convenciones de Tampa y Charlotte, puede servir para ilustrar el papel esencial desempeñado por el discurso emocional en la estrategia electoral de ambos candidatos.

\section{Método}

La presente investigación tiene como principal objetivo identificar los marcadores emocionales presentes en los textos pronunciados por las Primeras Damas en las convenciones demócrata y republicana en la campaña presidencial de 2012: Michelle Obama y Ann Romney. Evidenciaremos, así, las interrelaciones entre el pathos y el logos aristotélico, mostrando las estrategias retóricas que tienen que ver con la introducción de una carga emocional puestas en práctica en la construcción de los textos pronunciados por las Primeras Damas en apoyo a sus respectivos maridos.

Partimos de la hipótesis de que dichos recursos retóricas que enfatizan el carácter emocional de un discurso son utilizadas de un modo estratégico, integrándose en el marco de la estrategia de construcción de la imagen del político durante la campaña. De ese modo, su identificación y análisis permiten vislumbrar un perfil humano de cada candidato sustentado en una caracterización de atributos específicos sobre los que gira todo el discurso emocional.

Desde un punto de vista metodológico, nos valdremos de herramientas de análisis textual eminentemente cuantitativas (como Wordle y Textalyser), si bien complementaremos también el estudio con técnicas cualitativas de análisis del discurso.

La perspectiva adoptada es de corte principalmente semántico. Prestaremos, así, especial atención a los mecanismos de selección léxica que permiten la conformación de campos semánticos dentro de la unidad textual, pues el léxico se revela como factor decisivo a la hora de propiciar las interpretaciones realizadas por parte de la audiencia, al orientar y modelar la argumentación.

Con todo, en esta investigación también ofreceremos una aproximación a otros recursos lingüístico-textuales que favorecen la construcción del discurso emocional, considerando que los procedimientos del lenguaje que comportan carga emotiva -al menos, carga emotiva potencial- son de muy diversa naturaleza. 
Así, ante la heterogeneidad de instrumentos lingüísticos que permiten la representación textual de las emociones en el discurso, nuestro estudio se centrará en analizar sólo el valor expresivo de aquellos que más incidencia han mostrado en los discursos de Ann Romney y Michelle Obama. Además, dado que la emoción puede manifestarse en diversos planos y niveles discursivos (Amossy, 2000: 20), hemos optado por diferenciarlos también dentro del análisis.

De este modo, delimitaremos, tres niveles de análisis. En primer lugar, un plano semántico o de contenido en el que distinguiremos los campos semánticos prioritariamente activados en los discursos señalando los ítems léxicos que los conforman respectivamente y su índice de frecuencia en el discurso. En segundo lugar, dentro del plano formal, diferenciaremos el nivel morfosintáctico y en él analizaremos el uso de las anáforas y estructuras repetitivas, así como el empleo de diminutivos, superlativos y estructuras comparativas. Finalmente, hablaremos también del nivel pragmático y estableceremos cuál ha sido el uso de los deícticos dentro de cada uno de los textos, veremos cuál es la fórmula de tratamiento y referencia empleada para remitir a sus respectivos esposos y comprobaremos la incidencia de enunciados con modalidad exclamativa o interrogativa.

Como colofón al apartado de análisis, ofreceremos, asimismo, una síntesis de los procedimientos empleados por las Primeras Damas a la hora de configurar el perfil humano de los candidatos, aludiendo a los valores y atributos que estas les asignan para definir su imagen presidenciable ante el auditorio. Del mismo modo, detallaremos la repercusión obtenida por ambos discursos en las redes sociales.

\section{Análisis y Resultados}

A continuación, presentamos los datos extraídos del análisis de las distintas estrategias retóricas de orden semántico, morfosintáctico y pragmático puestas en práctica para facilitar la introducción en los discursos de las Primeras Damas de una carga emocional capaz de construir un perfil humano del candidato y de conmover al auditorio.

\subsection{Recursos semánticos utilizados en la construcción del relato emocional}

Dentro del plano estrictamente semántico, resulta especialmente relevante el uso de un léxico con marcada carga emocional como estrategia persuasiva. La revisión de las intervenciones de las esposas de los candidatos presidenciales permite establecer que ambas ofrecieron en sus discursos un repertorio de voces que se aglutinan en torno a tres grandes campos semánticos estrechamente vinculados entre sí: familia, sentimientos y sociopolítica (Anexo 2).

En total, Ann Romney empleó 535 veces ítems léxicos que pueden ser agrupados de acuerdo con estos subconjuntos semánticos en su discurso. Michelle Obama, por su parte, los utilizó en 603 ocasiones. Teniendo en cuenta que las voces más recurrentes dentro del discurso son las preposiciones, artículos, conjunciones y los pronombres, la frecuencia con que son usados resulta más que significativa en los dos textos ${ }^{2}$.

2 El discurso completo de Ann Romney tiene una extensión de 1355 palabras y el de Michelle Obama, de 1743. 
Es preciso matizar, sin embargo, que en ambos casos, según se aprecia en en la tabla 1 y en las figuras 1 y 2 (ver también Anexo 2), la cantidad de palabras destinadas a designar realidades o fenómenos sociopolíticos fue inferior a las voces capaces de expresar o suscitar emociones en el auditorio (un total de 384 de 535 y de 399 de 603 , respectivamente), con lo que puede establecerse que familia y sentimientos se erigieron en los dos grandes polos significativos de los discursos de Ann Romney y Michelle Obama.

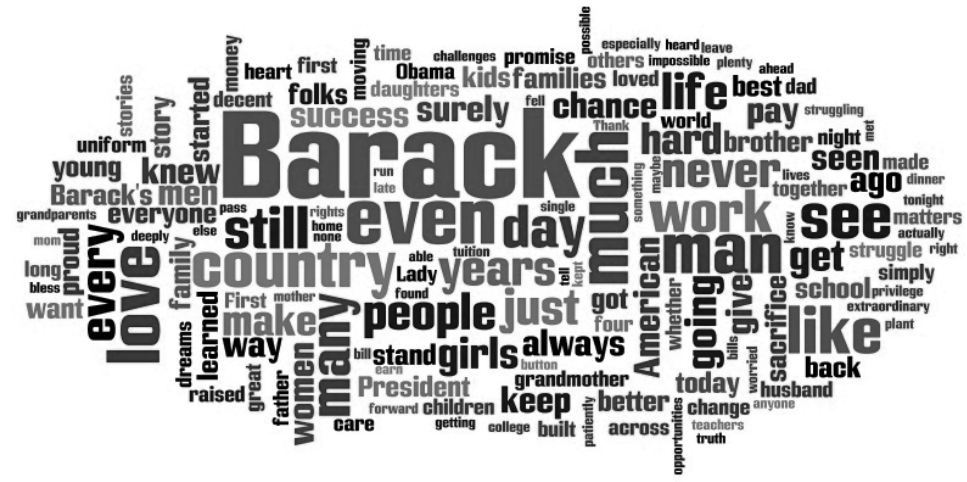

Figura 1. Tagcloud Michelle Obama. Fuente: Elaboración propia y Wordle.com

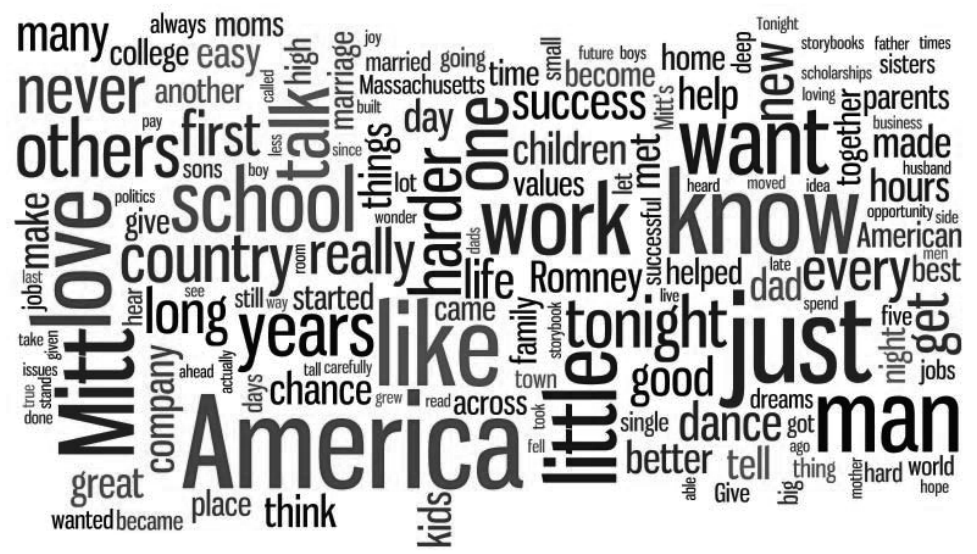

Figura 2. Tagcloud Ann Romney. Fuente: Elaboración propia y Wordle.com

Según se desprende de la lectura de los discursos de las Primeras Damas, las unidades léxicas relacionadas con familia y sentimientos se reforzaron estableciendo referencias cruzadas dentro del texto y fueron tejiendo, con ello, una red de isotopías semánticas $^{3}$ que permitieron articularlo como una unidad de sentido con marcado ca-

3 La noción de isotopía (Greimas, 1977) remite específicamente a la conexión o vínculo semántico subyacente que se establece entre las unidades léxicas que conforman el armazón textual con el fin de otorgarle sentido unitario y propiciar una interpretación desambiguada del mensaje por parte del destinatario. 
rácter emocional. En ocasiones, las oradoras expresaron sentimientos propios; otras veces emitieron enunciados en los que asignan sentimientos a una tercera persona -sus maridos, esencialmente-; además, también reprodujeron mensajes más complejos cuya única finalidad era conmover al público.

Tabla 1. Recursos semánticos: uso de lexemas pertenecientes a los campos semánticos Familia y Sentimientos/Emoción

\begin{tabular}{|c|c|c|c|}
\hline \multicolumn{4}{|c|}{ Recursos semánticos: uso de lexemas pertenecientes a los campos semánticos Familia y Sentimientos/Emoción } \\
\hline Michelle Obama & Total ítems & Tipología & Función \\
\hline & 399 & $\begin{array}{l}\text { - Voces que describen emociones. } \\
\text { (afraid, satisfaction, panic) } \\
\text { - Voces que suscitan emociones } \\
\text { (poverty, home, family). } \\
\text { - Enunciados complejos que evocan } \\
\text { emociones } \\
\text { ("I've seen it in the young man } \\
\text { blinded by a bomb in Afghanistan } \\
\text { who said, simply: "I'd give my eyes } \\
100 \text { times again to have the chance to } \\
\text { do what I have done and what I can } \\
\text { still do"). }\end{array}$ & $\begin{array}{l}\text { - Expresión de } \\
\text { sentimientos propios. } \\
\text { ("I love my husband } \\
\text { even more than I did } \\
\text { four years ago"). } \\
\text { - Asignación de } \\
\text { sentimientos a una } \\
\text { tercera persona } \\
\text { ("He wants everyone in } \\
\text { this country to have that } \\
\text { same opportunity"). }\end{array}$ \\
\hline Ann Romney & 384 & $\begin{array}{l}\text { - Voces que describen emociones. } \\
\text { (feel, complaint, love) } \\
\text { - Voces que suscitan emociones } \\
\text { (mom, illness, sacrifice). } \\
\text { - Enunciados complejos que evocan } \\
\text { emociones ("The single dad who's } \\
\text { working extra hours tonight, so that } \\
\text { his kids can buy some new clothes to } \\
\text { go back to school, can take a school } \\
\text { trip or play a sport, so his kids can } \\
\text { feel... like the other kids") }\end{array}$ & $\begin{array}{l}\text { - Expresión de } \\
\text { sentimientos propios. } \\
\text { ("I could tell you why I } \\
\text { fell in love with him") } \\
\text { - Asignación de } \\
\text { sentimientos a una } \\
\text { tercera persona } \\
\text { ("Mitt doesn't like to } \\
\text { talk about how he has } \\
\text { helped others") }\end{array}$ \\
\hline
\end{tabular}

\subsection{Recursos morfosintácticos utilizados en la construcción del relato emocional} Al servicio de la persuasión emocional las esposas de los candidatos articularon también en sus discursos otros mecanismos de carácter morfosintáctico o gramatical. Concretamente, resulta relevante la destacada presencia en ellos de anáforas y de estructuras repetitivas, así como el empleo frecuente de diminutivos, superlativos y fórmulas comparativas.

La anáfora consiste en la repetición de una o más palabras al inicio de varias secuencias sintácticas. Esta figura retórica, que posee un carácter enfatizante y propicia un efecto enaltecedor, adquirió especial relevancia dentro de los discursos de Michelle Obama -I've seen it in [...], I love, [...], if [...]- y Ann Romney, -"I want to talk to you [...]", "This man will" [...]".

Gracias a su acción, las posiciones y afirmaciones de las dos oradoras se vieron reforzadas, pues ayudó a generar una sensación de certidumbre y favoreció la dimensión estética de sus intervenciones. Tal como indican Cantavella et al. (2008: 86), "la repetición -empleada moderadamente y distribuida de forma uniforme- puede otorgar una función de seguridad y contundencia al discurso".

Por otra parte, otro recurso gramatical empleado por ambas que redundó en la emotividad de sus mensajes fue el uso de diminutivos, superlativos y estructuras compa- 
rativas, pues estos elementos comportan una carga subjetiva por parte del hablante y proporcionan un valor eminentemente afectivo.

El discurso de Ann Romney fue más prolijo en el empleo de "little" como diminutivo o atenuador expresivo ("a little intimidated", "a little less", "a little bit", "a little harder", "little things", etc.), mientras que Michelle Obama mostró mayor predilección por el empleo de comparativos y superlativos: "In the greatest nation on Earth", "Barack and I were doing what's best for our girls", "how hard you work matters more than how much you make, that helping others means more than just getting ahead yourself').

\subsection{Recursos pragmáticos utilizados en la construcción del relato emocional}

Finalmente, en el ámbito pragmático, los dispositivos activados en los discursos de las esposas de los candidatos en busca de la efectividad emotiva fueron, fundamentalmente, el uso de las modalidades interrogativa y exclamativa en sus enunciados, el empleo de deícticos y la utilización de apelativos cariñosos para hacer referencia a sus maridos.

La interrogación y la exclamación retórica se emplean habitualmente en los textos como vehículo de refuerzo o énfasis de una afirmación o negación previa y como mecanismo de intensificación de los sentimientos o estados de ánimo del hablante, sentimientos de los que el emisor quiere hacer partícipe también al destinatario del mensaje. Con todo, en nuestro caso, debemos advertir que es sobre todo la esposa del candidato republicano quien hizo mayor uso de estos dispositivos, pues Michelle Obama se mostró mucho más comedida a la hora de utilizarlos. Así, muy especialmente, Ann Romney empleó en su intervención la exclamación y interrogación retórica y, en particular, la interrogación retórica con implicatura afirmativa -la que se construye mediante partículas negativas-: It's how it is, isn't it? You know it's true, don't you?"Ann Romney; "How would we keep them grounded under the glare of the national spotlight?, Michelle Obama.

Por otro lado, la forma escogida prioritariamente por ambas para remitir a sus respectivos maridos también contribuyó a aportar el efecto de cercanía y proximidad en sus discursos. El empleo del nombre de pila del candidato de manera recurrente a lo largo de sus intervenciones reveló un trato mucho más personal y generó una sensación de familiaridad e intimidad en el auditorio.

Michelle Obama nombró 26 veces a su esposo utilizando el apelativo "Barack". Únicamente hizo uso del nombre y apellido completo -Barack Obama- en 3 ocasiones y se refirió a él como "my husband" en 5. Además, la Primera Dama también mencionó en una ocasión el hipocorístico con el que la abuela de Barack Obama suele llamarle cariñosamente: "Bar". Por su parte, las referencias de Ann Romney a su esposo se redujeron a 10 ocasiones con su nombre de pila y a 5 con el nombre completo -Mitt Romney-. La esposa del candidato republicano no alude a él en ningún momento tampoco mediante la construcción sintagmática "my husband".

Estos datos permiten establecer que en el discurso de Michelle Obama la figura del candidato presidencial adquirió mucha más relevancia y que la personalización y familiaridad a la hora de referirse al marido fue una estrategia de cercanía afectiva más explotada por la Primera Dama frente a la esposa del candidato republicano. 
En último lugar, la revisión del uso de deícticos dentro de los discursos de las esposas de los candidatos presidenciales también dejó entrever información relevante para la construcción del discurso emocional. Este tipo de elementos lingüísticos, que constituyen mecanismos de cohesión que facilitan la coherencia textual, permiten realizar una tarea de anclaje de los mensajes lingüísticos en el contexto específico en el que aparecen insertos.

En las alocuciones de Ann Romney y Michelle Obama, especial relevancia adquirieron los deícticos de carácter personal -pronombres personales y adjetivos determinativos posesivos- por su gran incidencia frente a los de naturaleza espacial o local. De acuerdo con los datos recopilados, los deícticos personales resultaron especialmente abundantes en ambos discursos (Michelle Obama presentó 366 formas pronominales personales; Ann Romney, 242).

El análisis de los textos revela que las dos esposas realizaron intervenciones en las que abundaron sobre todo las formas pronominales en primera y segunda persona $(59,2 \%$, Michelle Obama; $64 \%$, Ann Romney), lo que favoreció una mayor proximidad afectiva al discurso, pues, con ello, se remite a los sujetos participantes de la enunciación.

Las formas pronominales de segunda persona del plural (you, your, yourself) sirvieron para generar apelaciones directas al auditorio y permiten que éste se involucre directamente en el mensaje transmitido por el orador.

Mayor efecto de familiaridad y cercanía social generó, sin embargo, el uso de las formas pronominales en primera persona del plural (we, us, our etc.). Al interpelar a la audiencia por medio de estos pronombres, se creó un efecto de inclusión, pues se consigue aunar en una única entidad a emisor y receptores ${ }^{4}$.

Con todo, también fue abultada la presencia en los discursos de otros elementos lingüísticos en tercera persona del singular de género masculino (19,1\%, Michelle Obama; 20,6\%, Ann Romney). La utilización recurrente de las formas masculinas de tercera persona del singular se vincula específicamente con la presencia obligada en el discurso de marcas referenciales que remiten a sus respectivos maridos, aunque en alguna ocasión estas formas se asocian también con otros referentes masculinos mencionados en el texto (el padre de Michelle Obama, el padre de Ann Romney, el padre de Mitt Romney, etc.).

${ }^{4}$ Con todo, en el caso de Ann Romney, es preciso advertir que las formas pronominales en plural (you, we, us, your, etc.) no se dirigen a la colectividad social en su conjunto, sino a un grupo concreto de personas: las mujeres. La esposa del candidato republicano orienta su discurso específicamente al público femenino: "We're the mothers, we're the wives, we're the grandmothers, we're the big sisters, we're the little sisters, we're the daughters. 
Tabla 2. Cuadro resumen de recursos morfosintácticos y pragmáticos.

Fuente: elaboración propia.

\begin{tabular}{|c|c|c|}
\hline \multicolumn{3}{|c|}{ Recursos morfosintácticos } \\
\hline Tipología & Función & Ejemplos \\
\hline $\begin{array}{l}\text { Anáforas y } \\
\text { estructuras } \\
\text { repetitivas }\end{array}$ & $\begin{array}{l}\text { - Enfatización y enaltecimiento. } \\
\text { - Refuerzo en las afirmaciones: sensación de } \\
\text { certidumbre }\end{array}$ & 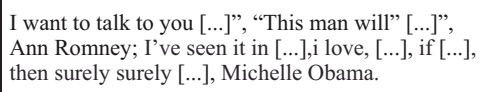 \\
\hline $\begin{array}{l}\text { Diminutivos } \\
\text { comparativos } \\
\text { superlativos }\end{array}$ & $\begin{array}{l}\text { - Transmisión de una carga subjetiva por parte del } \\
\text { hablante: transmisión de valores afectivos. } \\
\text { - Refuerzo enfático }\end{array}$ & $\begin{array}{l}\text { "little intimidated", "a little less", "a little bit" } \\
\text { "Barack and I were doing what's best for our girls", } \\
\text { "how hard you work matters more than how much } \\
\text { you make, that helping others means more than just } \\
\text { getting ahead yourself". }\end{array}$ \\
\hline \multicolumn{3}{|c|}{ Recursos pragmáticos } \\
\hline Tipología & Función & Ejemplos \\
\hline $\begin{array}{l}\text { Modalidad } \\
\text { interrogativa } \\
\text { exclamativa }\end{array}$ & $\begin{array}{l}\text { - Desarrollo de la función apelativa y expresiva del } \\
\text { lenguaje: interpelan al interlocutor y muestran la } \\
\text { subjetividad del hablante. }\end{array}$ & $\begin{array}{l}\text { It's how it is, isn't it? You know it's true, don't } \\
\text { you?", Ann Romney; "How would we keep them } \\
\text { grounded under the glare of the national spotlight?, } \\
\text { Michelle Obama. }\end{array}$ \\
\hline Deícticos & $\begin{array}{l}\text { Generación del efecto de cercanía y proximidad: } \\
\text { anclaje de los mensajes lingüísticos en el contexto } \\
\text { específico en el que aparecen insertos. }\end{array}$ & $\begin{array}{l}\text { Michelle Obama: } 366 \text { formas pronominales; Ann } \\
\text { Romney: } 242 \text {. Preferencia por las formas } \\
\text { pronominales en primera y segunda persona. }\end{array}$ \\
\hline $\begin{array}{l}\text { Nombre de pila del } \\
\text { candidato }\end{array}$ & $\begin{array}{l}\text { - Generación del efecto de cercanía y proximidad: trato } \\
\text { mucho más personal y genera una sensación de } \\
\text { familiaridad e intimidad en el auditorio }\end{array}$ & $\begin{array}{l}\text { Michelle Obama: } 26 \text { menciones con el nombre de } \\
\text { pila, } 3 \text { con el nombre completo y } 1 \text { con el } \\
\text { hipocorístico "Bar". } \\
\text { El número de referencias de Ann Romney: } 10 \\
\text { menciones con su nombre de pila y } 5 \text { con el nombre } \\
\text { completo. }\end{array}$ \\
\hline
\end{tabular}

\subsection{La construcción del perfil humano del candidato presidencial: atributos $\mathrm{y}$ valores}

En Tampa y Charlotte, Michelle Obama y Ann Romney debían cumplir con una misma misión: configurar el perfil humano del candidato presidencial y descubrir su lado más personal desde una mirada íntima con objeto de lograr conmover a la audiencia. La revisión de los textos dejó entrever, sin embargo, que el retrato que trazaron ambas esposas de los candidatos republicano y demócrata y el camino que siguieron para lograrlo difirió bastante en uno y otro caso, aunque emplearon, en ocasiones, recursos y estrategias similares.

Ambas contaron al auditorio cómo conocieron a sus maridos y les declararon su amor incondicional ${ }^{5}$. Además, también salpicaron sus alocuciones con anécdotas familiares e imágenes costumbristas en tono confidencial ${ }^{6}$ y aludieron, asimismo, a episodios especialmente dolorosos de sus vidas para generar la sensación de cercanía y provocar la compasión en el público (esclerosis múltiple del padre de Michelle Obama y cáncer de mama y esclerosis múltiple de Ann Romney).

5 "I'm still in love with that boy I met at a high school dance", Ann Romney; "I deeply loved the man I had built that life with [...] I loved Barack just the way he was", Michelle Obama.

6 "We walked to class together, shared the housekeeping, and ate a lot of pasta and tuna fish", Ann Romney; "Saturdays at soccer games, Sundays at grandma's house.... and a date night for Barack and me was either dinner or a movie", Michelle Obama. 
Otros puntos en común de la argumentación emocional llevada a cabo por ambas fue la alusión al sueño americano y la presentación de algunas figuras familiares como modelos y ejemplos a seguir. Estos personajes aparecieron ensalzados en el discurso y se les atribuyeron cualidades heroicas, cualidades que posteriormente se vincularon con el candidato presidencial. Ann Romney mencionó a dos figuras masculinas: el padre de Ann y el padre de Mitt Romney; Michelle Obama, por el contrario, señaló una figura masculina -el padre de Michelle- y otra femenina -la abuela de Barack Obama-.

Con todo, los atributos y valores asignados a ambos candidatos resultaron diferentes en ambos discursos. Michelle Obama establece una conexión indisoluble entre vida y política. De acuerdo con su argumentación, las experiencias vitales han influenciado y condicionado decisivamente la visión y la actuación política del presidente, de modo que en Barack Obama no hay separación posible entre el político y el hombre. Este vínculo le dibuja como una figura eminentemente empática que conoce y comprende los problemas de la ciudadanía ${ }^{7}$.Esta imagen se refuerza, a su vez, con la presentación que su esposa hace de él como hombre sencillo y corriente y como $^{8}$ hombre que encarna los valores de sacrificio, constancia, esfuerzo personal, modestia, dignidad, decencia, honestidad, integridad, gratitud y humildad atribuidos al padre de Michelle y a la abuela del presidente.

El binomio biografía-política no se explotó, sin embargo, en el discurso de Ann Romney con la misma sutileza ni con la misma intensidad argumentativa. La esposa del candidato republicano aportó una visión más pragmática y menos intimista y emotiva concentrándose sobre todo en la confianza como valor prioritario al retratar a su marido. Ann Romney presentó, así, a su esposo como un hombre de éxito en los negocios muy trabajador y como un candidato fiable y resolutivo capaz de sacar a América de la difícil situación económica que atraviesa, mencionando para ello los logros empresariales de Mitt Romney y los buenos resultados políticos cosechados en Massachusetts cuando él era gobernador'.

\subsection{Valoración del seguimiento y la resonancia de ambos discursos en las redes sociales}

Para finalizar este análisis, conviene apuntar algunos datos sobre la repercusión que sus locuciones tuvieron sobre el electorado americano, a partir de la diferente resonancia que tuvieron en las redes sociales.

Pese a que los especialistas coinciden en que el perfil del usuario de Twitter no se puede generalizar al votante medio de los Estados Unidos, lo cierto es que la plataforma de microblogging se convirtió en un medidor del clima electoral. Así, Michelle Obama

\footnotetext{
7 "Because Barack knows what it means, he knows what it means", Michelle Obama.

8 "Even though back then Barack was a Senator and a presidential candidate to me... [...] he was the guy whose proudest possession was a coffee table he'd found in a dumpster, and whose only pair of decent shoes was half a size too small", Michelle Obama.

9 "This man will not fail. This man will not let us down. This man will lift up America! [...] You can trust Mitt. He loves America. He will take us to a better place", Ann Romney.
} 
se convirtió en una tendencia mundial en la red social durante su discurso. Tanto ella (@MichelleObama) como la convención tuvieron 3 millones de menciones bajo el hashtag \#DNC2012, de acuerdo con el mismo blog de Twitter. Se registraron 28.003 publicaciones por minuto, comparados con los 6.000 tuits por minuto de Ann Romney.

En Facebook, la Primera Dama también ganó la batalla en lo que se refiere a repercusión pública. Una de las frases de su discurso que más sonaron fue: "He visto de primera mano que ser presidente no cambia quién eres... Revela quién eres". Una imagen de la Primera Dama con la leyenda de esa frase, compartida desde su cuenta de Facebook recibió cerca de 100 mil likes y más de 2.500 comentarios.

La gran repercusión del discurso de Michelle Obama también quedó demostrada en el gráfico comparativo realizado por el Washington Post que evidencia la popularidad de la Primera Dama sobre la esposa del candidato republicano, a partir del número de búsquedas en Google, durante sus respectivos discursos. Tal y como refleja la figura 3, considerando el nivel de interés de búsquedas en torno a ambas generado durante ambos discursos, Michelle Obama ganó la batalla a Ana Romney. La mujer del candidato demócrata no sólo obtuvo mayor número de búsquedas durante el comienzo de su discurso, sino que, además, ésta diferencia fue considerablemente mayor al finalizar su discurso, tal y como se aprecia en el gráfico.

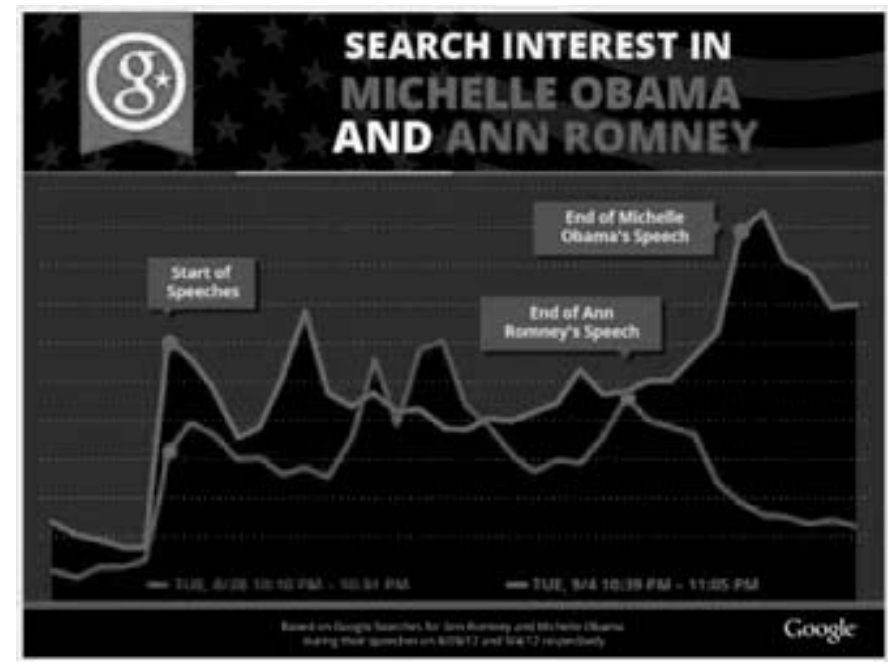

Figura 3. Búsquedas en Google durante los discursos de Michelle Obama y Ann Romney. Fuente: Google.

\section{Conclusiones}

La instrumentalización política de las emociones ha sido una herramienta que se ha revelado especialmente eficaz en el marco de la comunicación política desde sus inicios debido a la gran rentabilidad persuasiva mostrada en el proceso de seducción del auditorio.

En EEUU, la esposa del candidato es una figura clave que mejor encarna esta conexión indisoluble entre política y emociones, pues su labor esencial es contribuir a construir el perfil humano del candidato mostrando su lado más íntimo y personal. 
Esta investigación ha analizado los discursos pronunciados por Ann Romney y Michelle Obama en las convenciones republicana y demócrata que tuvieron lugar en la campaña presidencial de Estados Unidos de 2012. El análisis de los recursos lingüísticos utilizados en ambos discursos desvela que estos se articularon con una marcada orientación emocional y se mostraron intencionadamente despolitizados: las dos aspirantes a Primeras Damas situaron en gran medida sus discursos fuera de las coordenadas temáticas de la agenda política estadounidense y construyeron un relato emocional focalizando la atención en el interés humano y personal de sus respectivos maridos.

La emoción, que constituye el eje central sobre el que gravita la configuración del discurso de las esposas de los candidatos, se canaliza, como hemos comprobado, a través de distintos dispositivos lingüístico-textuales. En este estudio, concretamente, nos hemos fijado en evidenciar la carga emotiva de algunos recursos de orden semántico, de carácter morfosintáctico y de naturaleza pragmática.

La información extraída del análisis cuantitativo y cualitativo que se ha llevado a cabo revela que las esposas de los candidatos configuraron sus intervenciones en torno a tres polos significativos: emoción y sentimientos, familia y sociopolítica. La activación en el discurso de ítems léxicos relacionados con estos campos semánticos permite generar isotopías semánticas que otorgan coherencia a la unidad textual y que son capaces de activar los resortes emocionales del público por la presencia de un gran número de voces que expresan o suscitan sentimientos.

Tal como se detalla en el estudio, en los relatos de Ann Romney y Michelle Obama también operaron otros recursos de índole morfosintáctica y pragmática que contribuyeron a facilitar la persuasión emocional, aportando mayor cercanía y expresividad al discurso. Específicamente, hemos comprobado la importancia adquirida por ciertos mecanismos, como el uso de diminutivos, superlativos, comparativos y de estructuras repetitivas, y de otros recursos, como el empleo del nombre de pila de los candidatos, el uso de las modalidades interrogativa y exclamativa y la utilización de deícticos.

Por otro lado, el análisis realizado reveló asimismo que en la conformación del perfil humano de Mitt Romney y Barack Obama, las esposas de los aspirantes a la presidencia de EEUU insistieron también en la argumentación emocional. Ambas coincidieron en construir estratégicamente un relato sobre la figura de sus respectivos maridos sustentando en los valores familiares más aceptados y presentaron a sus maridos como personas inteligentes, trabajadoras, buenas y bien intencionadas. Esta imagen positiva trataba de inducir en la audiencia emociones que movilizaran el voto, al influir de manera favorable en los juicios en torno a las figuras de los presidenciables.

Con todo, los datos recopilados apuntaron también algunas diferencias en la estrategia. Así, Michelle Obama estableció un binomio biografía-política sobre Barack Obama, con el que proyectó ante el auditorio una imagen de su marido como una figura empática capaz de comprender y entener las necesidades del ciudadano americano. El retrato personal e intimista que realizó Michelle sobre Barack Obama contrastó con la visión más pragmática y política que Ann Romney imprimió al per- 
fil de Mitt Romney, pues el candidato republicano aparecía definido más bien en términos de eficiencia política y empresarial, a través de la imagen de un político fiable capaz de solventar los problemas de América.

Estas divergencias fueron quizá decisivas a la hora de inclinar la balanza en favor de Michelle Obama en el seguimiento y la repercusión de ambos discursos en las redes sociales. A tenor de los datos estadísticos manejados sobre su presencia en Internet, Twitter y Facebook la esposa del candidato demócrata construyó un discurso más cercano y próximo a la ciudadanía, pues logró una mayor conexión con el público.

En definitiva, el estudio realizado muestra cómo tanto Ann Romney como Michelle Obama, a través de sus discursos, se erigieron en bisagras emocionales que vincularon al candidato con el electorado y cómo ello las convirtió en piezas clave en la estrategia electoral desarrollada por sus maridos en la carrera por llegar a la Casa Blanca durante la campaña electoral de 2012. Este estudio da, así, un paso más en la comprobación empírica del importante papel que va adquiriendo la figura de la Primera Dama en la estrategia política electoral, especialmente, en lo que respecta al fortalecimiento de la dimensión más humana del liderazgo político, y es este un aspecto sobre el que habrá que prestar más atención en las futuras campañas electorales.

\section{Referencias bibliográficas}

AMOSSY, Ruth (2006): L'argumentation dans le discours. Paris, Armand Colin.

ARROYAS, Enrique; PÉREZ, Pedro Luis; y BERNÁ, Celia (2012): "Del des-contexto al descontento: agenda y discurso de la prensa durante las elecciones autonómicas en la Región de Murcia (2011)". Estudios sobre el Mensaje Periodístico, Vol. 18, doi: http://dx.doi.org/10.5209/rev_ESMP.2012.v18.40891. Madrid, Servicio de Publicaciones de la Editorial Complutense, pp. 87-99.

BURNS, Lisa M. (2005): “Collective Memory and the Candidates' Wives in the 2004 Presidential Campaign". Rhetoric \& Public Affairs, $\mathrm{n}^{\circ} 8$ (4), pp. 684-688.

BRADER, Ted (2011): “Arousing Public Passions”. En HOFINGER, Christophe; MANZ-CHRIST, Gerlinde: Emotions in politics and Campaigning: How Neuroscience, Linguistics, and Social Psychology Change the Political Profession. Nueva Delhi, Prestige Books International, pp. 38-54.

CANTAVELLA, Juan; BULLOUGH, Rachel; CURIEL, Luis Antonio; MORALES, Begoña; MEJÍA, César; y PITTARO, Esteban (2008): "Algunos aspectos lingüísticos de los debates electorales Zapatero-Rajoy 2008". Estudios sobre el Mensaje Periodístico, Vol. 14, doi: inf/ 11341629/articulos/ESMP0808110079A.PDF. Madrid, Servicio de Publicaciones de la Editorial Complutense, pp. 79-98.

CAROLI, Betty Boyd (2003): First Ladies: From Martha Washington to Laura Bush. New York, Oxford University Press.

CHARAUDEAU, Patrick (2000): “Une problématique discursive de l'émotion. À propos des effets de pathémisation à la télévision". En PLANTIN, Christian; DOURY, Marianne; y TRAVERSO, Véronique (eds.): Les émotions dans les interactions, Lyon, Presses universitaires de Lyon, pp. 125-155. 
Rocío Zamora Medina et al. La retórica emocional de la esposa del candidato: análisis lingüistico...

GREIMAS, Algirdas (1977): Semántica estructural. Madrid, Gredos.

GRIMES, Ann (1990): Running Mates: The Making of a First Lady. New York, William Marrow \& \& Co.

GOOTY, Janaki; CONNELLY, Shane; GRIFFITH, Jennifer; y GUPTA, Alka (2010): "Leadership, affect and emotions: A state of the science review". The Leadership Quarterly, nº 21 (6), pp. 979-1004.

GUTIÉRREZ-RUBÍ, Antoni (2012): Elecciones USA 2012: los 12 factores decisivos. (ebook), en http://www.gutierrez-rubi.es/elecciones-usa-2012-los-12-factores-decisivos/ [fecha de consulta: 15/05/2013]

HOFINGER, Christophe; MANZ-CHRIST, Gerlinde (2011): Emotions in politics and Campaigning: How Neuroscience, Linguistics, and Social Psychology Change the Political Profession. Nueva Delhi, Prestige Books International.

KERBRAT-ORECCHIONI, Catherine (2000): "Quelle place pour les émotions dans la linguistique du XXe siècle? Remarques et aperçus". En PLANTIN, Christian; DOURY, Marianne; y TRAVERSO, Véronique (eds.): Les émotions dans les interactions, Lyon, Presses Universitaires de Lyon, pp. 33-74.

LUNTZ, Frank (2007): Words That Work: It's Not What You Say, It's What People Hear. New York, Hyperion.

MACMANUS, Susan y QUECAN, Andrew (2008): "Spouses as Campaign Surrogates: Strategic Appearances by Presidential and Vice Presidential Candidates Wives in the 2004 Election". Wives in the 2004 Election. PS: Political Science \&amp; Politics, $\mathrm{n}^{\circ}$ 41, pp. 337-348.

MORGANTE, Jole (2010): “Communication politique et passion”. Altre modernità: Rivista di studi letterari e culturali, $\mathrm{n}^{\mathrm{O}} 3$, pp. 18-26.

PUIG, Luisa (2008): "Del pathos clásico al análisis del discurso". Acta Poética no 29 (2), pp. 393-413.

RAJAH, Rashimah; SONG, Zaholi; y ARVEY, Richard D. (2011): "Emotionality and leadership: taking stock of the past decade of research". The Leadership Quarterly, no 22 (6): pp. 1107-1119.

REDLAWSK, David (2006): "Feeling politics: new research into emotion and politics". En REDLAWSK, David (ed.): Feeling Politics: Emotion in Political Information Processing, Nueva York, Palgrave Macmillan, pp. 1-10.

VANHORN, Ann Marie (2010): “Candidate Wives: Spouses as Strategic Surrogates on the Presidential Campaign Trail" (epub), en: http://docs.lib.purdue.edu/dissertations/AAI3453394/ [fecha de consulta: 22/05/2013]

WATSON, Robert P. (2000): The Presidents' wives: reassessing the office of First Lady. Boulder, Colorado, Lynne Rienner Publishers.

WERTHEIMER, Molly Meijer (ed., 2005): Leading Ladies of the White House: Communication Strategies of Notable Twentieth-Century First Ladies. Boulder, Colorado, Rowman \& Littlefield Publishers. 
WESTEN, Drew (2007): The Political Brain: The Role of Emotion in Deciding the Fate of the Nation. New York, Public Affairs.

WINFIELD, Betty Houchin \& FRIEDMAN, Barbara (2003): "Gender Politics: News Coverage of the Candidates' Wives in Campaign 2000". Journalism and Mass Communication Quarterly, $\mathrm{n}^{\circ} 80$ (3), pp. 548-566.

ZACCARO, Stephen J. (2012): "Individual differences and leadership: Contributions to a third tipping point". The Leadership Quarterly, n² 23(4), pp. 718-728.

\section{Anexo 1: Discursos de Ann Romney y Michelle Obama 8.1. Discurso íntegro de Ann Romney en Tampa}

Luce, thank you for that kind introduction.

I want to talk to you tonight not about politics and not about party.

And while there are many important issues we'll hear discussed in this convention and throughout this campaign, tonight I want to talk to you from my heart about our hearts.

I want to talk not about what divides us, but what holds us together as an American family. I want to talk to you tonight about that one great thing that unites us, that one thing that brings us our greatest joy when times are good, and the deepest solace in our dark hours.

Tonight I want to talk to you about love.

I want to talk to you about the deep and abiding love I have for a man I met at a dance many years ago. And the profound love I have, and I know we share, for this country.

I want to talk to you about that love so deep only a mother can fathom it - the love we have for our children and our children's children.

And I want us to think tonight about the love we all share for those Americans, our brothers and sisters, who are going through difficult times, whose days are never easy, nights are always long, and whose work never seems done.

They are here among us tonight in this hall; they are here in neighborhoods across Tampa and all across America. The parents who lie awake at night side by side, wondering how they'll be able to pay the mortgage or make the rent; the single dad who's working extra hours tonight, so that his kids can buy some new clothes to go back to school, can take a school trip or play a sport, so his kids can feel... like the other kids.

And the working moms who love their jobs but would like to work just a little less to spend more time with the kids, but that's just out of the question with this economy. Or that couple who would like to have another child, but wonder how will they afford it.

I've been all across this country for the past year and a half and heard these stories of how hard it is to get ahead now. I've heard your voices: "I'm running in place," "we just can't get ahead."

Sometimes I think that late at night, if we were all silent for just a few moments and listened carefully, we could hear a great collective sigh from the moms and dads across America who made it through another day, and know that they'll make it through another one tomorrow. But in that end of the day moment, they just aren't sure how.

And if you listen carefully, you'll hear the women sighing a little bit more than the men. It's how it is, isn't it?

It's the moms who always have to work a little harder, to make everything right. 
It's the moms of this nation - single, married, widowed - who really hold this country together. We're the mothers, we're the wives, we're the grandmothers, we're the big sisters, we're the little sisters, we're the daughters.

You know it's true, don't you?

You're the ones who always have to do a little more.

You know what it's like to work a little harder during the day to earn the respect you deserve at work and then come home to help with that book report which just has to be done.

You know what those late night phone calls with an elderly parent are like and the long weekend drives just to see how they're doing.

You know the fastest route to the local emergency room and which doctors actually answer the phone when you call at night.

You know what it's like to sit in that graduation ceremony and wonder how it was that so many long days turned into years that went by so quickly.

You are the best of America.

You are the hope of America.

There would not be an America without you.

Tonight, we salute you and sing your praises.

I'm not sure if men really understand this, but I don't think there's a woman in America who really expects her life to be easy. In our own ways, we all know better!

And that's fine. We don't want easy. But these last few years have been harder than they needed to be. It's all the little things - that price at the pump you just can't believe, the grocery bills that just get bigger; all those things that used to be free, like school sports, are now one more bill to pay. It's all the little things that pile up to become big things. And the big things - the good jobs, the chance at college, that home you want to buy, just get harder. Everything has become harder.

We're too smart to know there aren't easy answers. But we're not dumb enough to accept that there aren't better answers.

And that is where this boy I met at a high school dance comes in.

His name is Mitt Romney and you really should get to know him.

I could tell you why I fell in love with him - he was tall, laughed a lot, was nervous - girls like that, it shows the guy's a little intimidated - and he was nice to my parents but he was really glad when my parents weren't around.

That's a good thing. And he made me laugh.

I am the granddaughter of a Welsh coal miner who was determined that his kids get out of the mines. My dad got his first job when he was six years old, in a little village in Wales called Nantyffyllon, cleaning bottles at the Colliers Arms.

When he was 15, dad came to America. In our country, he saw hope and an opportunity to escape from poverty. He moved to a small town in the great state of Michigan. There, he started a business - one he built himself, by the way.

He raised a family. And he became mayor of our town.

My dad would often remind my brothers and me how fortunate we were to grow up in a place like America. He wanted us to have every opportunity that came with life in this country - and so he pushed us to be our best and give our all. 
Inside the houses that lined the streets of our town, there were a lot of good fathers teaching their sons and daughters those same values. I didn't know it at the time, but one of those dads was my future father-in-law, George Romney.

Mitt's dad never graduated from college. Instead, he became a carpenter.

He worked hard, and he became the head of a car company, and then the governor of Michigan.

When Mitt and I met and fell in love, we were determined not to let anything stand in the way of our life together. I was an Episcopalian. He was a Mormon.

We were very young. Both still in college. There were many reasons to delay marriage, and you know? We just didn't care. We got married and moved into a basement apartment. We walked to class together, shared the housekeeping, and ate a lot of pasta and tuna fish. Our desk was a door propped up on sawhorses. Our dining room table was a fold down ironing board in the kitchen. Those were very special days.

Then our first son came along. All at once I'm 22 years old, with a baby and a husband who's going to business school and law school at the same time, and I can tell you, probably like every other girl who finds herself in a new life far from family and friends, with a new baby and a new husband, that it dawned on me that I had absolutely no idea what I was getting into.

That was 42 years ago. Now we have five sons and 18 grandchildren and I'm still in love with that boy I met at a high school dance.

I read somewhere that Mitt and I have a "storybook marriage". Well, in the storybooks I read, there were never long, long, rainy winter afternoons in a house with five boys screaming at once. And those storybooks never seemed to have chapters called MS or Breast Cancer.

A storybook marriage? No, not at all. What Mitt Romney and I have is a real marriage.

I know this good and decent man for what he is - warm and loving and patient.

He has tried to live his life with a set of values centred on family, faith, and love of one's fellow man. From the time we were first married, I've seen him spend countless hours helping others. I've seen him drop everything to help a friend in trouble, and been there when latenight calls of panic came from a member of our church whose child had been taken to the hospital.

You may not agree with Mitt's positions on issues or his politics. Massachusetts is only 13\% Republican, so it's not like that's a shock.

But let me say this to every American who is thinking about who should be our next President:

No one will work harder.

No one will care more.

No one will move heaven and earth like Mitt Romney to make this country a better place to live!

It's true that Mitt has been successful at each new challenge he has taken on. It amazes me to see his history of success actually being attacked. Are those really the values that made our country great? As a mom of five boys, do we want to raise our children to be afraid of success?

Do we send our children out in the world with the advice, "Try to do...OK?"

And let's be honest. If the last four years had been more successful, do we really think there would be this attack on Mitt Romney's success?

Of course not. 
Mitt will be the first to tell you that he is the most fortunate man in the world. He had two loving parents who gave him strong values and taught him the value of work. He had the chance to get the education his father never had.

But as his partner on this amazing journey, I can tell you Mitt Romney was not handed success.

He built it.

He stayed in Massachusetts after graduate school and got a job. I saw the long hours that started with that first job. I was there when he and a small group of friends talked about starting a new company. I was there when they struggled and wondered if the whole idea just wasn't going to work. Mitt's reaction was to work harder and press on.

Today that company has become another great American success story.

Has it made those who started the company successful beyond their dreams?

Yes, it has.

It allowed us to give our sons the chance at good educations and made all those long hours of book reports and homework worth every minute. It's given us the deep satisfaction of being able to help others in ways that we could never have imagined. Mitt doesn't like to talk about how he has helped others because he sees it as a privilege, not a political talking point. And we're no different than the millions of Americans who quietly help their neighbours, their churches and their communities. They don't do it so that others will think more of them.

They do it because there IS no greater joy.

"Give and it shall be given unto you."

But because this is America, that small company which grew has helped so many others lead better lives. The jobs that grew from the risks they took have become college educations, first homes. That success has helped fund scholarships, pensions, and retirement funds. This is the genius of America: dreams fulfilled help others launch new dreams.

At every turn in his life, this man I met at a high school dance, has helped lift up others. He did it with the Olympics, when many wanted to give up.

He did it in Massachusetts, where he guided a state from economic crisis to unemployment of just $4.7 \%$.

Under Mitt, Massachusetts's schools were the best in the nation. The best. He started the John and Abigail Adams scholarships, which give the top $25 \%$ of high school graduates a fouryear tuition-free scholarship.

This is the man America needs.

This is the man who will wake up every day with the determination to solve the problems that others say can't be solved, to fix what others say is beyond repair. This is the man who will work harder than anyone so that we can work a little less hard.

I can't tell you what will happen over the next four years. But I can only stand here tonight, as a wife, a mother, a grandmother, an American, and make you this solemn commitment:

This man will not fail.

This man will not let us down.

This man will lift up America!

It has been 47 years since that tall, kind of charming young man brought me home from our first dance. Not every day since has been easy. 
But he still makes me laugh. And never once did I have a single reason to doubt that I was the luckiest woman in the world.

I said tonight I wanted to talk to you about love. Look into your hearts.

This is our country.

This is our future.

These are our children and grandchildren.

You can trust Mitt.

He loves America.

He will take us to a better place, just as he took me home safely from that dance.

Give him that chance.

Give America that chance.

God bless each of you and God Bless the United States of America.

\subsection{Discurso íntegro de Michelle Obama en Charlotte}

Thank you so much, Elaine...we are so grateful for your family's service and sacrifice.... and we will always have your back.

Over the past few years as First Lady, I have had the extraordinary privilege of traveling all across this country.

And everywhere I've gone, in the people I've met, and the stories I've heard, I have seen the very best of the American spirit.

I have seen it in the incredible kindness and warmth that people have shown me and my family, especially our girls.

I've seen it in teachers in a near-bankrupt school district who vowed to keep teaching without pay.

I've seen it in people who become heroes at a moment's notice, diving into harm's way to save others...flying across the country to put out a fire...driving for hours to bail out a flooded town.

And I've seen it in our men and women in uniform and our proud military families...in wounded warriors who tell me they're not just going to walk again, they're going to run, and they're going to run marathons... in the young man blinded by a bomb in Afghanistan who said, simply, "...I'd give my eyes 100 times again to have the chance to do what I have done and what I can still do."

Every day, the people I meet inspire me...every day, they make me proud...every day they remind me how blessed we are to live in the greatest nation on earth.

Serving as your First Lady is an honor and a privilege... but back when we first came together four years ago, I still had some concerns about this journey we'd begun.

While I believed deeply in my husband's vision for this country... and I was certain he would make an extraordinary President...like any mother, I was worried about what it would mean for our girls if he got that chance.

How would we keep them grounded under the glare of the national spotlight?

How would they feel being uprooted from their school, their friends, and the only home they'd ever known?

Our life before moving to Washington was filled with simple joys...Saturdays at soccer games, Sundays at grandma's house... and a date night for Barack and me was either dinner or a movie, because as an exhausted mom, I couldn't stay awake for both. 
And the truth is, I loved the life we had built for our girls...I deeply loved the man I had built that life with... and I didn't want that to change if he became President.

I loved Barack just the way he was.

You see, even though back then Barack was a Senator and a presidential candidate...to me, he was still the guy who'd picked me up for our dates in a car that was so rusted out, I could actually see the pavement going by through a hole in the passenger side door... he was the guy whose proudest possession was a coffee table he' $d$ found in a dumpster, and whose only pair of decent shoes was half a size too small.

But when Barack started telling me about his family - that's when I knew I had found a kindred spirit, someone whose values and upbringing were so much like mine.

You see, Barack and I were both raised by families who didn't have much in the way of money or material possessions but who had given us something far more valuable - their unconditional love, their unflinching sacrifice, and the chance to go places they had never imagined for themselves.

My father was a pump operator at the city water plant, and he was diagnosed with Multiple Sclerosis when my brother and I were young.

And even as a kid, I knew there were plenty of days when he was in pain...I knew there were plenty of mornings when it was a struggle for him to simply get out of bed.

But every morning, I watched my father wake up with a smile, grab his walker, prop himself up against the bathroom sink, and slowly shave and button his uniform.

And when he returned home after a long day's work, my brother and I would stand at the top of the stairs to our little apartment, patiently waiting to greet him...watching as he reached down to lift one leg, and then the other, to slowly climb his way into our arms.

But despite these challenges, my dad hardly ever missed a day of work...he and my mom were determined to give me and my brother the kind of education they could only dream of.

And when my brother and I finally made it to college, nearly all of our tuition came from student loans and grants.

But my dad still had to pay a tiny portion of that tuition himself.

And every semester, he was determined to pay that bill right on time, even taking out loans when he fell short.

He was so proud to be sending his kids to college... and he made sure we never missed a registration deadline because his check was late.

You see, for my dad, that's what it meant to be a man.

Like so many of us, that was the measure of his success in life - being able to earn a decent living that allowed him to support his family.

And as I got to know Barack, I realized that even though he'd grown up all the way across the country, he'd been brought up just like me.

Barack was raised by a single mother who struggled to pay the bills, and by grandparents who stepped in when she needed help.

Barack's grandmother started out as a secretary at a community bank... and she moved quickly up the ranks...but like so many women, she hit a glass ceiling.

And for years, men no more qualified than she was - men she had actually trained - were promoted up the ladder ahead of her, earning more and more money while Barack's family continued to scrape by. 
But day after day, she kept on waking up at dawn to catch the bus... arriving at work before anyone else...giving her best without complaint or regret.

And she would often tell Barack, "So long as you kids do well, Bar, that's all that really matters."

Like so many American families, our families weren't asking for much.

They didn't begrudge anyone else's success or care that others had much more than they did...in fact, they admired it.

They simply believed in that fundamental American promise that, even if you don't start out with much, if you work hard and do what you're supposed to do, then you should be able to build a decent life for yourself and an even better life for your kids and grandkids.

That's how they raised us...that's what we learned from their example.

We learned about dignity and decency - that how hard you work matters more than how much you make...that helping others means more than just getting ahead yourself.

We learned about honesty and integrity - that the truth matters... that you don't take shortcuts or play by your own set of rules... and success doesn't count unless you earn it fair and square.

We learned about gratitude and humility - that so many people had a hand in our success, from the teachers who inspired us to the janitors who kept our school clean....and we were taught to value everyone's contribution and treat everyone with respect.

Those are the values Barack and I - and so many of you - are trying to pass on to our own children.

That's who we are.

And standing before you four years ago, I knew that I didn't want any of that to change if Barack became President.

Well, today, after so many struggles and triumphs and moments that have tested my husband in ways I never could have imagined, I have seen firsthand that being president doesn't change who you are - it reveals who you are.

You see, I've gotten to see up close and personal what being president really looks like.

And I've seen how the issues that come across a President's desk are always the hard ones - the problems where no amount of data or numbers will get you to the right answer...the judgment calls where the stakes are so high, and there is no margin for error.

And as President, you can get all kinds of advice from all kinds of people.

But at the end of the day, when it comes time to make that decision, as President, all you have to guide you are your values, and your vision, and the life experiences that make you who you are.

So when it comes to rebuilding our economy, Barack is thinking about folks like my dad and like his grandmother.

He's thinking about the pride that comes from a hard day's work.

That's why he signed the Lilly Ledbetter Fair Pay Act to help women get equal pay for equal work.

That's why he cut taxes for working families and small businesses and fought to get the auto industry back on its feet.

That's how he brought our economy from the brink of collapse to creating jobs again jobs you can raise a family on, good jobs right here in the United States of America. 
When it comes to the health of our families, Barack refused to listen to all those folks who told him to leave health reform for another day, another president.

He didn't care whether it was the easy thing to do politically - that's not how he was raised - he cared that it was the right thing to do.

He did it because he believes that here in America, our grandparents should be able to afford their medicine... our kids should be able to see a doctor when they're sick... and no one in this country should ever go broke because of an accident or illness.

And he believes that women are more than capable of making our own choices about our bodies and our health care...that's what my husband stands for.

When it comes to giving our kids the education they deserve, Barack knows that like me and like so many of you, he never could've attended college without financial aid.

And believe it or not, when we were first married, our combined monthly student loan bills were actually higher than our mortgage.

We were so young, so in love, and so in debt.

That's why Barack has fought so hard to increase student aid and keep interest rates down, because he wants every young person to fulfill their promise and be able to attend college without a mountain of debt.

So in the end, for Barack, these issues aren't political - they're personal.

Because Barack knows what it means when a family struggles.

He knows what it means to want something more for your kids and grandkids.

Barack knows the American Dream because he's lived it... and he wants everyone in this country to have that same opportunity, no matter who we are, or where we're from, or what we look like, or who we love.

And he believes that when you've worked hard, and done well, and walked through that doorway of opportunity...you do not slam it shut behind you...you reach back, and you give other folks the same chances that helped you succeed.

So when people ask me whether being in the White House has changed my husband, I can honestly say that when it comes to his character, and his convictions, and his heart, Barack Obama is still the same man I fell in love with all those years ago.

He's the same man who started his career by turning down high paying jobs and instead working in struggling neighborhoods where a steel plant had shut down, fighting to rebuild those communities and get folks back to work...because for Barack, success isn't about how much money you make, it's about the difference you make in people's lives.

He's the same man who, when our girls were first born, would anxiously check their cribs every few minutes to ensure they were still breathing, proudly showing them off to everyone we knew.

That's the man who sits down with me and our girls for dinner nearly every night, patiently answering their questions about issues in the news, and strategizing about middle school friendships.

That's the man I see in those quiet moments late at night, hunched over his desk, poring over the letters people have sent him.

The letter from the father struggling to pay his bills...from the woman dying of cancer whose insurance company won't cover her care... from the young person with so much promise but so few opportunities. 
I see the concern in his eyes...and I hear the determination in his voice as he tells me, "You won't believe what these folks are going through, Michelle...it's not right. We've got to keep working to fix this. We've got so much more to do."

I see how those stories - our collection of struggles and hopes and dreams - I see how that's what drives Barack Obama every single day.

And I didn't think it was possible, but today, I love my husband even more than I did four years ago...even more than I did 23 years ago, when we first met.

I love that he's never forgotten how he started.

I love that we can trust Barack to do what he says he's going to do, even when it's hard especially when it's hard.

I love that for Barack, there is no such thing as "us" and "them" - he doesn't care whether you're a Democrat, a Republican, or none of the above... he knows that we all love our country... and he's always ready to listen to good ideas... he's always looking for the very best in everyone he meets.

And I love that even in the toughest moments, when we're all sweating it - when we're worried that the bill won't pass, and it seems like all is lost - Barack never lets himself get distracted by the chatter and the noise.

Just like his grandmother, he just keeps getting up and moving forward... with patience and wisdom, and courage and grace.

And he reminds me that we are playing a long game here... and that change is hard, and change is slow, and it never happens all at once.

But eventually we get there, we always do.

We get there because of folks like my Dad...folks like Barack's grandmother...men and women who said to themselves, "I may not have a chance to fulfill my dreams, but maybe my children will...maybe my grandchildren will."

So many of us stand here tonight because of their sacrifice, and longing, and steadfast love... because time and again, they swallowed their fears and doubts and did what was hard.

So today, when the challenges we face start to seem overwhelming - or even impossible let us never forget that doing the impossible is the history of this nation...it's who we are as Americans...it's how this country was built.

And if our parents and grandparents could toil and struggle for us...if they could raise beams of steel to the sky, send a man to the moon, and connect the world with the touch of a button...then surely we can keep on sacrificing and building for our own kids and grandkids.

And if so many brave men and women could wear our country's uniform and sacrifice their lives for our most fundamental rights...then surely we can do our part as citizens of this great democracy to exercise those rights...surely, we can get to the polls and make our voices heard on Election Day.

If farmers and blacksmiths could win independence from an empire...if immigrants could leave behind everything they knew for a better life on our shores... if women could be dragged to jail for seeking the vote...if a generation could defeat a depression, and define greatness for all time...if a young preacher could lift us to the mountaintop with his righteous dream....and if proud Americans can be who they are and boldly stand at the altar with who they love...then surely, surely we can give everyone in this country a fair chance at that great American Dream.

Because in the end, more than anything else, that is the story of this country - the story of unwavering hope grounded in unyielding struggle. 
That is what has made my story, and Barack's story, and so many other American stories possible.

And I say all of this tonight not just as First Lady... and not just as a wife.

You see, at the end of the day, my most important title is still "mom-in-chief."

My daughters are still the heart of my heart and the center of my world.

But today, I have none of those worries from four years ago about whether Barack and I were doing what's best for our girls.

Because today, I know from experience that if I truly want to leave a better world for my daughters, and all our sons and daughters... if we want to give all our children a foundation for their dreams and opportunities worthy of their promise... if we want to give them that sense of limitless possibility - that belief that here in America, there is always something better out there if you're willing to work for it...then we must work like never before... and we must once again come together and stand together for the man we can trust to keep moving this great country forward...my husband, our President, President Barack Obama.

Thank you, God bless you, and God bless America.

\section{Anexo 2: Campos semánticos en los discursos de Ann Romney y Michelle Obama: unidades léxicas y frecuencias}

\begin{tabular}{|c|c|c|}
\hline \multicolumn{3}{|c|}{ Ann Romney } \\
\hline Campo semántico Sentimientos y emociones & Campo semántico Familia & Campo semántico Sociopolítica \\
\hline $\begin{array}{l}\text { Afraid (1), amaze (1), amazing (1) become (7), best } \\
\text { (4), better (4), bless (2), cancer (1), care (2), } \\
\text { carefully (2), challenge (1), chance (3), charming } \\
\text { (1), commitment (1), determination (1), determined } \\
\text { (2), dreams (3), expect (1), faith (1), feel (1), fell in } \\
\text { love (2), fortunate (2), friends (2), get ahead (2), } \\
\text { glad (1), good (6), great (5), heart, (3), help ( } 9) \text {, } \\
\text { honest (1), hope (2), imagine (1), intimidated (1), } \\
\text { joy (2), kind (2), laugh (2), Life (6), like (vbo.) (3), } \\
\text { live (5), love (sust.) (7), love (vbo.) (3), ms } \\
\text { (multiple sclerosis) (1), need (sust.) (1), need (vbo.) } \\
\text { (1), nervous, nice (1), ooportunity (2), panic (1), } \\
\text { patient (1), poverty } \\
\text { (1), praise (1), pushed (1), quietly } \\
\text { (1), reaction (1), respect (1), satisfaction (1), scream } \\
\text { (1), share (3), side by side (1), sighing (1), solace } \\
\text { (1), storybook (3), struggle } \\
\text { (1), success (6), successful (3), thank (1), together } \\
\text { (4) trouble (1), trust (1), unite (1), values(4), want } \\
\text { (13), warm (1) }\end{array}$ & $\begin{array}{l}\text { Boy (2), brother (2), child (8) couple (1) dad } \\
\text { (7), daugther (2) elderly parents (1), family } \\
\text { (4), } \\
\text { father (3), girl (2), grandchildren (2), } \\
\text { grandaughter (1), grandmother (1), grocery } \\
\text { bill (1), grow up (3), home (6), homework } \\
\text { (1), house } \\
\text { (2) housekeeping (1), husband (2), kids (5), } \\
\text { kitchen (1), marriage (4), married (5), moms } \\
\text { (5), mother (1), neighborhoods (1), } \\
\text { neighbours (1), parents (4), partner (1), raise } \\
\text { (2), single (3), sister (3), son (3), widowed } \\
\text { (1), wife (2) }\end{array}$ & $\begin{array}{l}\text { America (12), American (4), Americans (2), } \\
\text { business (2) } \\
\text { campaign (1), collage } \\
\text { (4), company (5), country (7), crisis (1), } \\
\text { doctors (1), economy (1), education (3), } \\
\text { governor (1), graduate (3), graduation (1), } \\
\text { high school (4), hospital (1), job (6), man (14), } \\
\text { nation, (2) party (1), pension (1), political (1), } \\
\text { politics (2), President (1), price (1), rent (1), } \\
\text { republican (1), retirement (1), scholarship (3), } \\
\text { school (7), state (2), tuition (1), woman (1), } \\
\text { work (sust.) (2), work (vbo.) (9), working } \\
\text { (adj.) (1) }\end{array}$ \\
\hline
\end{tabular}




\begin{tabular}{|c|c|c|}
\hline \multicolumn{3}{|c|}{ Michelle Obama } \\
\hline $\begin{array}{l}\text { Admire (1), aid (2), begrudge (1), better (2), bless } \\
\text { (2), brave, (1), cancer (1), care (4), challenge } \\
\text { (2), chance (6), change (5) } \\
\text { complaint (1), concern (vbo) } \\
\text { (2), courage (1), decency (1) decent (3), depression } \\
\text { (1), determination (1), determined } \\
\text { (2), dignity (1), dreams (2) dye (1), fear (1), feel (1) } \\
\text { fell in love } \\
\text { (1) friendship, (1) friends (2) } \\
\text { joy (1), Get ahead (2)good (2) grateful (1) gratitude } \\
\text { (1), great (2) harm (1), help (sust.) (2) } \\
\text { help (vbo) (2), heroe (1), honestly } \\
\text { (1), honesty, (1), humility (1), illness (1), } \\
\text { impossible } \\
\text { (2), incredible (1) inspire (2), joy (1), kindness (1), } \\
\text { kind (2), life (8), like (2), live (5), love (sust.) (3) } \\
\text { love (11), Multiple sclerosis (1), } \\
\text { pain (1), patience (1) } \\
\text { patiently (2), personal (2), possible (2), pride (1), } \\
\text { privilege (2), problems (1), promise (3), proud (4), } \\
\text { quiet (1), regret (1), respect (1), sacrifice (1), sick } \\
\text { (1), smile (1), spirit, (2) struggle (sust.), } \\
\text { (3), struggle (vbo) (5), success (5), triumph (1), } \\
\text { trust (2), truth (2), togeher (3) unconditional (1), } \\
\text { unflinching (1), unwavering, (1), unyielding (1), } \\
\text { valuable (1), value (2), want (5), warmth (1), } \\
\text { worried (adj.) (2) worry (sust.) (1), wounded (1) }\end{array}$ & $\begin{array}{l}\text { Brother (4), be born (1), children (2), } \\
\text { community (2), crib (1), dad (5), daughter } \\
\text { (3), dinner (2), family (13), father (3), } \\
\text { friendship (1), friends (1) girls (4), } \\
\text { grandchildren (1), grandkids (3), grandma } \\
\text { (1), grandmother (3), grandparents (3), } \\
\text { grounded (2), grow up (1), home (2), house, } \\
\text { (2), husband (4), kids (7), kindred (1), marry } \\
\text { (1), mom (3), mortgage (1), mother (2), } \\
\text { neighborhood (1), parents (1), raised (2), rent } \\
\text { (1), single (1), son (1), upbringing (1), wife, } \\
\text { (1) }\end{array}$ & $\begin{array}{l}\text { America (2), American (6) } \\
\text { Americans (2), bank (1) } \\
\text { bankrupt (1), Bill (4), businesses } \\
\text { (1), candidate (1), citizens (1), city (1), collage } \\
\text { (3) conviction } \\
\text { (1) country (14), debt (2), } \\
\text { Democrat (1), earn (3) economy } \\
\text { (2), education (2), election day } \\
\text { (1), equal, (2) finantial (1), first lady (2), folks } \\
\text { (6), grant } \\
\text { (1), health (3), immigrans (1), jobs (4), man } \\
\text { (13), money (3), } \\
\text { national (1), nation (2), people } \\
\text { (9) political (1), politically (1) } \\
\text { polls (1), presidential (1), President (9), } \\
\text { registration } \\
\text { (1), Republican (1), right (sust.) } \\
\text { (2), school (4), senador (1), student loan (2), } \\
\text { tax(1), teachers } \\
\text { (2), tuition (2), vote (1), woman (7), work } \\
\text { (sust.) (6), work (vbo.) } \\
\text { (7) working (adj.) (1) }\end{array}$ \\
\hline
\end{tabular}

